\title{
LATE RESULTS AFTER CEMENT BONE FILLING IN TREATMENT OF GIANT CELL TUMOUR (GCT) - RETROSPECTIVE STUDY
}

doi: $10.2478 /$ rojost-2018-0040

\author{
R. Prejbeanu, D. Crişan, A. Bălănescu, H. Haraguş, B. Deleanu \\ "Victor Babeş" University of Medicine and Pharmacy, Timişoara, Romania
}

Introduction. Segmental or intralesional excision with curettage or complete resection can be one method of treatment for giant cell tumor (GCT), but the ideal filling material after curettage or resection remains controversial. The purpose of this retrospective study was to follow the latest results and complications regarding the recurrence or degradation of functional status that underwent cementation.

Material and methods. We reported 24 cases with GCTs during the last 15 years. All the patients were treated by intralesional excision or segmental resection followed by acrylic cement filling with or no metal augmentation recurrence-free survival proportions were used to evaluate oncological outcomes. Other parameters including surgical complication, general condition, and radiological classification were analyzed.

Results. We followed up 20 cases for at least five years postoperatory (from the $5^{\text {th }}$ to the $9^{\text {th }}$ year). The recurrence-free survival proportions showed that the recurrence rate in this group was for 4 patients ( 2 of them were at second surgery). 2 patients had degradation of implant cement fixation. Parameters including patients' age, gender, tumor location, and radiological classification did not affect the surgeons' treatments in cavity filling after GCT curettage.

Conclusions. Cementation should be recommended because of easy usage, have long lasting better results, and the better local tumor control than other methods (ex: bone grafting). The risk of recurrence is low and is not related to the cementation and metal augmentation.

The cost-benefit is also in favor of this technique.

Keywords: cementation, giant cell tumor, knee 